\title{
A MICROMACHINED PIEZOELECTRIC MICROPHONE FOR AEROACOUSTICS APPLICATIONS
}

\author{
Stephen Horowitz, Toshikazu Nishida, Louis Cattafesta, and Mark Sheplak ${ }^{\dagger}$
}

\author{
Interdisciplinary Microsystems Group, PO Box 116250, University of Florida, Gainesville, Florida 32611-6250 \\ †(352) 392-3983, FAX: (352) 392-7303, sheplak@ufl.edu
}

\begin{abstract}
This paper describes the design, fabrication, and characterization of a bulk-micromachined piezoelectric microphone for aeroacoustic applications. The microphone was fabricated by combining a sol-gel lead zirconate-titanate (PZT) deposition process on a silicon-on-insulator (SOI) wafer with deepreactive ion-etching (DRIE) for the diaphragm release. Experimental characterization indicates a sensitivity of $0.75 \mu \mathrm{V} / \mathrm{Pa}$, a dynamic range greater than six orders of magnitude $(47.8-169 \mathrm{~dB}, \mathrm{ref} .20 \mu \mathrm{Pa})$, a capacitance of
\end{abstract} $13.9 \mathrm{nF}$ and a resonant frequency of $50.8 \mathrm{kHz}$.

\section{INTRODUCTION}

The goal of this research is to develop a microphone for aeroacoustic wind-tunnel testing [1]. Microelectromechanical systems (MEMS)-based microphones have been developed utilizing piezoresistive, optical, capacitive, and piezoelectric transduction techniques [2-8]. Most of these designs focused on audio applications that typically require a maximum sound pressure level (SPL) of $\sim 120 d B$, integrated noise floors of $\leq 35 \mathrm{dBA}$ and bandwidths of $\leq 20 \mathrm{kHz}$. Conversely, aeroacoustics applications may require maximum sound pressure levels in excess of $160 \mathrm{~dB}$, bandwidths of $\geq 50 \mathrm{kHz}$, but the noise floor requirements are generally less stringent $(<50 \mathrm{~dB} / \sqrt{\mathrm{Hz}})$ than audio applications. Each transduction technique has unique advantages and disadvantages for aeroacoustic applications. Piezoelectric transduction offers the potential for a durable, high sensitivity, low noise device that requires no external power to operate. The main drawback is material integration and compatibility with standard micromachining.

A number of authors have developed piezoelectric microphones and have fabricated them using a wide array of piezoelectric materials $[\mathbf{2 - 6 , 8 , 9 ]}$. Thin film, piezoelectric materials include zinc oxide $(\mathrm{ZnO})$, aluminum nitride (AIN), aromatic polyurea, polyvinylidene fluoride (PVDF), and PZT. They each offer different mechanical and electrical properties, as well as transduction capabilities. In addition, some of the materials are CMOS compatible while others are not, which has led to many differing approaches to the implementation of piezoelectric microphones. The ease of integrating the piezoelectric material into a fabrication sequence is an important consideration in the development of a microphone. PZT, for instance, contains lead, barring it from all CMOS facilities, whereas AIN is fully CMOS compatible. $\mathrm{ZnO}$ and PVDF, while not fully CMOS compatible, can be integrated more easily with conventional processing. Despite the various microphones that utilize piezoelectric transduction, to date, no piezoelectric microphones have been developed specifically for aeroacoustic applications.

\section{SENSOR DESIGN}

This microphone was designed by combining a fundamental piezoelectric composite plate model with a lumped-element, electroacoustic model [10-11]. Lumped-element modeling (LEM) was used to estimate the theoretical sensitivity and bandwidth of the device. Finite element modeling was then used to verify the LEM predictions prior to fabrication.

In micromachined piezoelectric devices, thin ferroelectric films are deposited on substrates comprised of a composite of silicon, metals and oxides. Large in-plane residual stresses often result because of the fabrication-induced stresses, different thermal expansion coefficients between the films, and packaging effects. These in-plane stresses can dominate the mechanics of these devices and must be accounted for during design. For this microphone, the electromechanical design employed smalldeflection plate theory for the multi-layer piezoelectric diaphragm subjected to in-plane stresses, transverse pressure, and transverse electric field [11].

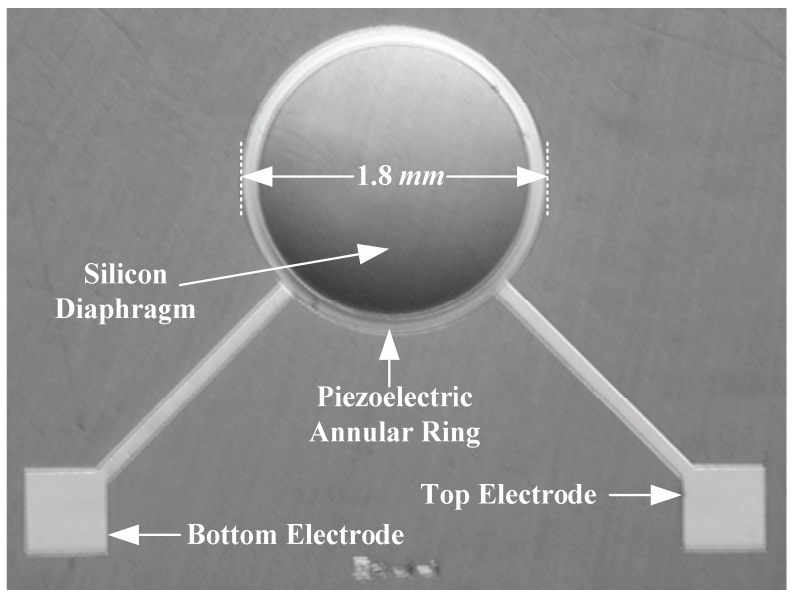

Figure 1: An optical photograph of the piezoelectric microphone.

The microphone structure consists of a $1.80 \mathrm{~mm}$ diameter, $3 \mu \mathrm{m}$ thick, silicon diaphragm with a thin ring of PZT placed near the boundary of the diaphragm (Figure 1 and Figure 2). The $1.85 \mathrm{~mm}$ diameter, $95 \mu \mathrm{m}$ wide, piezoelectric annular ring was placed near the diaphragm edge to maximize sensitivity due to higher stress concentrations in this region. The placement of the ring near the boundary also eliminates the need to run electrodes across the diaphragm. The $270 \mathrm{~nm}$ thick PZT was sandwiched between a $200 \mathrm{~nm}$ Ti/Pt layer and a $180 \mathrm{~nm}$ Pt layer that serve as bottom and top electrodes, respectively. The entire ring structure is separated from the silicon diaphragm by a $100 \mathrm{~nm}$ thick $\mathrm{TiO}_{2}$ layer that serves as a diffusion barrier for the PZT during processing. Inclusion of this diffusion barrier prevents cracking of the PZT that would otherwise arise from loss of lead via diffusion into the silicon during later processing steps. 


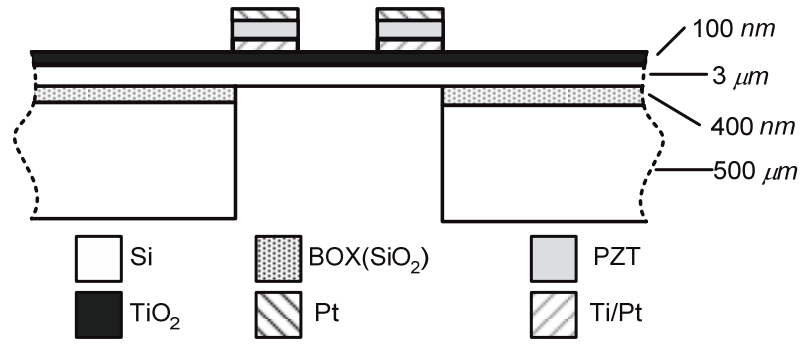

Figure 2: Cross sectional schematic showing silicon diaphragm with circular piezoelectric composite ring. (Not to scale.)

The transversely-isotropic composite plate model accounts for material properties of each of the thin-film layers, as shown in Figure 3. These include the mechanical properties of the layers such as Young's Modulus, $E$, density, $\rho$, and Poisson's ratio, $v$, and electrical properties including the relative permittivity constant, $\varepsilon_{r}$, and piezoelectric coefficient, $d_{31}$, of the PZT. The model also accounts for the in-plane compressive stress, $\sigma_{0}$, of each layer. Furthermore, the geometry of the structure is accounted for via the inner PZT radius, $R_{1}$, outer PZT radius, $R_{2}$, and the various film thicknesses. The material properties used for the electromechanical model are given in Table 1.

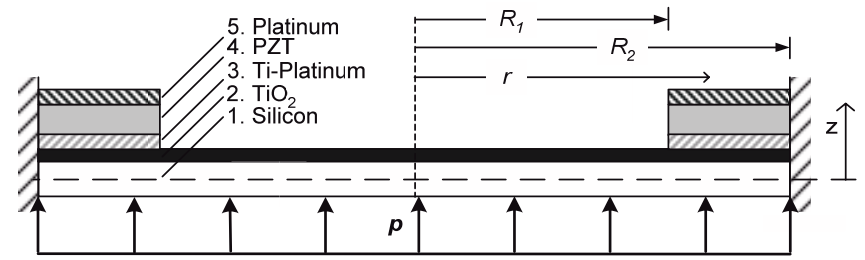

Figure 3: Cross-sectional schematic of the piezoelectric composite circular plate model.

Table 1: Material properties used for design.

\begin{tabular}{|c|c|c|c|c|c|c|}
\hline & $\begin{array}{c}d_{31} \\
{[p C / N]}\end{array}$ & $v$ & $\varepsilon_{r}$ & $\begin{array}{c}E \\
{[G P a]}\end{array}$ & $\begin{array}{c}\rho \\
{\left[\mathrm{kg} / \mathrm{m}^{3}\right]}\end{array}$ & $\begin{array}{c}\sigma_{0} \\
{[M P a]}\end{array}$ \\
\hline silicon & - & 0.3 & - & 169 & 2.5 & 0 \\
\hline $\mathrm{Pt} / \mathrm{Ti}$ & - & 0.38 & - & 170 & 21.44 & - \\
\hline $\mathrm{TiO}_{2}$ & - & 0.28 & - & 283 & 2.15 & 1809 \\
\hline $\mathrm{PZT}[12]$ & -50 & 0.3 & 500 & 30 & 7.5 & 176.0 \\
\hline
\end{tabular}

A lumped element model was then developed to facilitate design of the complete microphone system which involves electrical, mechanical, and acoustical energy domains. In LEM, the coupling between the various energy domains is realized via equivalent two-port models of the physical system [10]. An equivalent circuit model is constructed by lumping the distributed energy storage and dissipation into ideal, generalized one-port circuit elements. In an electroacoustic system, differential pressure and voltage are effort variables, while volumetric flow rate and current are flow variables. The lumped elements can then be represented in an equivalent electrical circuit by equating acoustic and mechanical elements to their electrical equivalents. In the electrical domain, a resistor represents dissipation of energy, while inductors and capacitors represent storage of kinetic and potential energy, respectively. Similarly, in the mechanical domain, kinetic and potential energy are stored in mass and compliance (inverse of stiffness), respectively. The same technique of lumping elements and equating them to their electrical equivalent can also be extended to the acoustical energy domain, thereby permitting a single equivalent circuit that represents the dynamics of the entire system. In this paper, an impedance analogy is employed, in which elements that share a common effort are connected in parallel, while those sharing a common flow are connected in series [10].

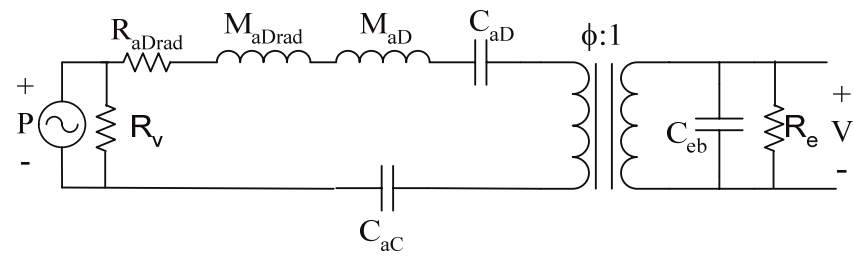

Figure 4: Equivalent circuit representation of a lumped-element model of a piezoelectric microphone.

Figure 4 shows a schematic of an equivalent circuit representation of the lumped element model where $R_{a D R a d}, M_{a D R a d}$, $C_{a D}$ and $M_{a D}$ are the radiation resistance and mass, and diaphragm compliance and mass, respectively. Also, $\phi, \mathrm{C}_{\mathrm{eb}}$ and $\mathrm{R}_{\mathrm{e}}$ are the electroacoustic transduction coefficient, blocked electrical capacitance and dielectric loss resistance, respectively. Finally, $C_{a c}$ is the cavity compliance and $R_{v}$ is the vent resistance. The structure of the equivalent circuit is explained as follows. An acoustic pressure, $P$, drives the motion of the diaphragm, which in turn generates a voltage, $V$, across the piezoelectric thin film represented by $C_{e b}$. This represents a conversion from the acoustical to the electrical domain and is accounted for via a transformer possessing a turns ratio

$$
\phi=\frac{-d_{A}}{C_{a s}} ;\left[\frac{P a}{V}\right],
$$

where $d_{A}$ is the piezoelectric coefficient and $C_{a D}$ is the diaphragm acoustic compliance when an electrical short circuit is imposed across the electrodes. The time-harmonic, two-port network equations describing the behavior of the piezoelectric transducer are written in the acoustic domain as

$$
\left[\begin{array}{l}
I \\
Q
\end{array}\right]=\left[\begin{array}{cc}
j \omega C_{e f} & j \omega d_{A} \\
j \omega d_{A} & j \omega C_{a D}
\end{array}\right]\left[\begin{array}{l}
V \\
P
\end{array}\right],
$$

where, $Q=\int \vec{V}(r) \cdot d \vec{S}$ is the volume velocity of the plate, $\vec{V}(r)=\frac{d w(r, t)}{d t}$ is the distributed velocity over the surface, $d \vec{S}$ is normal projection of an infinitesimal element of area, $w(r, t)$ is the transverse deflection, and $I$ is the electrical current [11]. The effective acoustic piezoelectric coefficient that relates the volumetric displacement, $\Delta \forall$, to the applied voltage for zero pressure loading is

$$
d_{A}=\left.\frac{\Delta \forall}{V}\right|_{P \rightarrow 0}=\frac{\left.\int_{0}^{R_{2}} w(r)\right|_{P \rightarrow 0} 2 \pi r d r}{V} ;\left[\frac{m^{3}}{V}\right],
$$

where $\Delta \forall=\int_{0}^{R_{2}} 2 \pi r w(r) d r=\int Q d t ;\left[m^{3}\right] . \quad$ Similiarly, $C_{a D}$ is defined by the volume displaced by the diaphragm for an 
applied pressure under zero voltage loading,

$$
C_{a D}=\left.\frac{\Delta \forall}{P}\right|_{V \rightarrow 0}=\frac{\left.\int_{0}^{R_{2}} w(r)\right|_{V \rightarrow 0} 2 \pi r d r}{P} ;\left[\frac{m^{3}}{P a}\right] .
$$

On the electrical side of the transformer, $C_{e b}$ is the blocked electrical capacitance and $R_{e}$ is the resistance of the piezoelectric ring. The term 'blocked' is used since it is the impedance seen by the source when the diaphragm motion is prevented. At frequencies greater than zero, an equivalent acoustic mass must also be included in the model as

$$
M_{a D}=2 \pi \int_{0}^{R_{2}} \rho_{A}\left(\frac{\left.w_{\text {inc }}(r)\right|_{V=0}}{\left.\Delta V\right|_{V=0}}\right)^{2} r d r ;\left[\frac{k g}{m^{4}}\right],
$$

where $\rho_{A}$ is the areal density of the piezoelectric composite plate given by

$$
\rho_{A}=\int_{z_{1}}^{z_{2}} \rho_{l} d z ;\left[\frac{k g}{m^{2}}\right]
$$

and $\rho_{l}$ is the density of the corresponding layer. Furthermore, this mass must be in series with the compliance as both possess the same motion (i.e., displacement or velocity)

Since the clamped circular plate is vibrating in a medium, the radiation impedance of the plate is also taken into account and consists of a radiation mass, $M_{a D r a d}$, and a radiation resistance, $R_{a \text { rad }}$, as seen in the circuit of Figure 4 . The radiation mass accounts for the inertial mass of the fluid that is vibrating in unison with the plate, while the radiation resistance accounts for the acoustic radiation of energy away from the plate. The radiation impedance can be found by modeling the structure as a piston in an infinite baffle. For $k R_{2}<<1$, the radiation resistance and mass can be approximated as [13]

$$
R_{a \text { rad }} \approx \frac{\left(k R_{2}\right)^{2} \rho c}{2 A_{e f f}} ;\left[\frac{k g}{m^{4} s}\right]
$$

and

$$
M_{a \operatorname{rad}} \approx \frac{8 k R_{2} \rho c}{3 \pi \omega A_{\text {eff }}} ;\left[\frac{\mathrm{kg}}{\mathrm{m}^{4}}\right],
$$

respectively, where $k=\omega / c$ is the wavenumber, $\omega$ is the angular frequency, $c$ is the isentropic speed of sound, and $A_{\text {eff }}$ is an effective diaphragm area to maintain continuity of volume velocity. Note the effects of structural damping terms could be added by putting a resistor in series with $R_{a D r a d}$. Additionally, the compliance of the cavity, $C_{a C}$, formed between the back of the diaphragm and the microphone package will affect the dynamic response and is therefore included in the equivalent circuit. The cavity compliance is defined as

$$
C_{a C}=\frac{\forall_{c}}{\rho c^{2}} ;\left[\frac{m^{3}}{P a}\right],
$$

where $\forall_{c}$ is the volume of the cavity. Finally, the resistance, $R_{v}$, represents viscous losses incurred in a vent channel that typically connects a microphone cavity to ambient pressure. In the actual device described in this paper, a vent channel was not specifically designed into the structure; however packaging-induced leakage between the cavity and atmospheric pressure provides the same effect.

An important point to note from the equivalent circuit is the effect that the cavity compliance, $C_{a C}$, can have on the overall sensitivity. If $C_{a C}$ is much larger than the diaphragm compliance, $C_{a D}$, then the sensitivity will not be noticeably affected. However, if the cavity compliance is the same order of magnitude or smaller than $C_{a D}$, then it will have a noticeable stiffening effect because $C_{a C}$ and $C_{a D}$ are in series. In order to avoid this generally undesirable effect, the cavity must be made large enough to have a much greater compliance than the diaphragm.

In order to determine most of the lumped element parameters, the distributed deflection, $w(r)$ must be determined.

To obtain $w(r)$, an analytical model was developed for the piezoelectric composite circular plate by Wang et al. [11]. Analytical modeling was accomplished by dividing the problem of Figure 3 into two portions, an inner circular plate surrounded by an annular composite ring with matching boundary conditions at the interface. The boundary conditions consist of equal moments and forces at the interface as well as equal slope and radial displacement. After solving for the deflection in each region, the deflection equation for each region was then combined into a single equation [11].

\section{FABRICATION}

The device fabrication is illustrated in Figures 5-8, highlighting the major steps in the process flow. The devices were fabricated using a sol-gel PZT deposition process on a 4-inch SOI wafer combined with DRIE for the release of the silicon diaphragm. The first step was to deposit a $100 \mathrm{~nm}$ thick layer of Ti which is then oxidized in a tube furnace to create a $\mathrm{TiO}_{2}$ layer that serves as a diffusion barrier [14] as shown in Figure 5a. A liftoff process was then used to pattern the bottom electrodes with $40 \mathrm{~nm}$ of evaporated Ti followed by $180 \mathrm{~nm}$ of evaporated Pt as shown in Figure $5 \mathrm{~b}$. The Ti layer served as an adhesion layer in this step.

a)
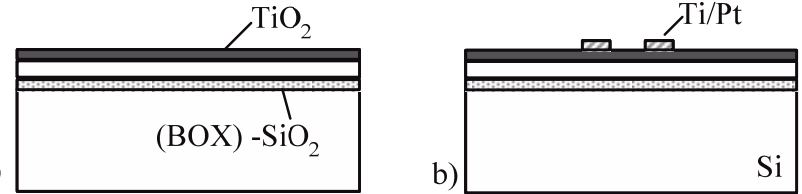

Figure 5: Initial process steps. a) Deposit $100 \mathrm{~nm}$ of Ti and oxidize to form $\mathrm{TiO}_{2}$. b) Deposit and liftoff Ti/Pt $\{40 / 180 \mathrm{~nm}\}$ to form bottom electrode.

The next step was the deposition of a previously mixed 52/48 sol-gel solution of PZT as shown in Figure 6a. The solution was spin-cast at $2500 \mathrm{RPM}$ for $30 \mathrm{sec}$, then pyrolized at $350{ }^{\circ} \mathrm{C}$ in air for $2 \mathrm{~min}$ on a hot plate. Repeating the spin and pyrolize step 6 times yielded a total PZT thickness of approximately $400 \mathrm{~nm}$. The wafers were then furnace annealed at $650{ }^{\circ} \mathrm{C}$ for $30 \mathrm{~min}$ to achieve a perovskite phase of PZT for the piezoelectric properties.

Following the PZT deposition, the top electrodes were deposited and patterned in a liftoff process similar to the bottom electrodes as shown in Figure 6b. The top electrodes also served as a hard etch mask for the PZT etch. The PZT was etched using a 3:1:1 solution of ammonium biflouride $\left(\left(\mathrm{NH}_{4}\right) \mathrm{HF}_{2}\right)$, hydrochloric acid $(\mathrm{HCl})$, and deionized water as shown in Figure $7 \mathrm{a}$. The etchant leaves a residue that then must be removed with a dilute 
nitric acid and hydrogen peroxide solution. The next step was to deposit a thick photoresist (PR) on the backside, and pattern the backside release etch as in Figure $7 \mathrm{~b}$.
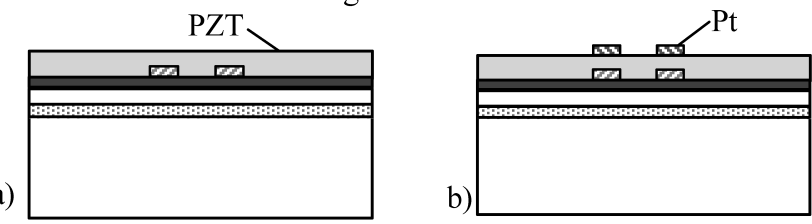

Figure 6: Continuing process steps. a) Spin PZT 52/48 \& pyrolize \{4 layers for $267 \mathrm{~nm}$ total\}. b) Deposit and liftoff Pt $\{180 \mathrm{~nm}\}$.

a)
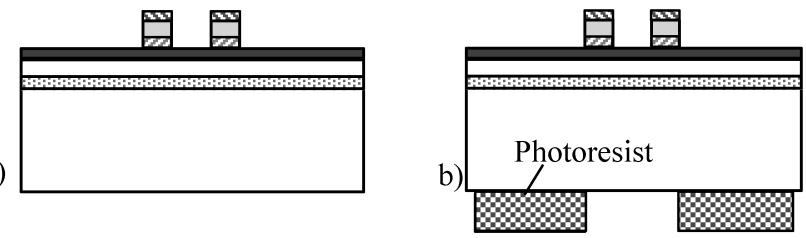

Figure 7: Continuing process steps. a) Wet etch PZT in 3:1:1 of $\left(\mathrm{NH}_{4}\right) \mathrm{HF}_{2} / \mathrm{HCl} / \mathrm{DI}$ water. b) Spin \& pattern thick PR on back.

Then the silicon wafer is backside etched using a DRIE process as shown in Figure 8a, stopping on the buried oxide (BOX) layer. Finally, the resist was ashed and the backside was wet etched in BOE to remove the exposed BOX.
国

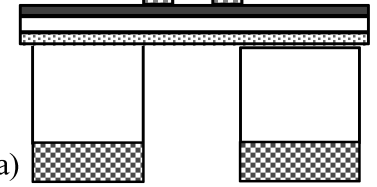

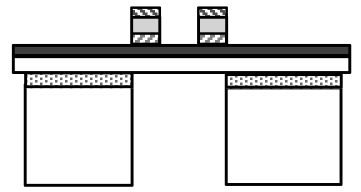

b)
Figure 8: Final process steps, device release. a) DRIE to BOX. b) Ash resist and wet etch backside to remove the $B O X$.

The chip was flush mounted in a Lucite package shown in Figure 9, using epoxy to secure it. The electrical connections to the package leads were accomplished via short lengths of bare wire that were bonded using silver epoxy on the chip bond pads as well as to the copper pads on the package. The completed packaged microphone is shown in the photograph of Figure 10.

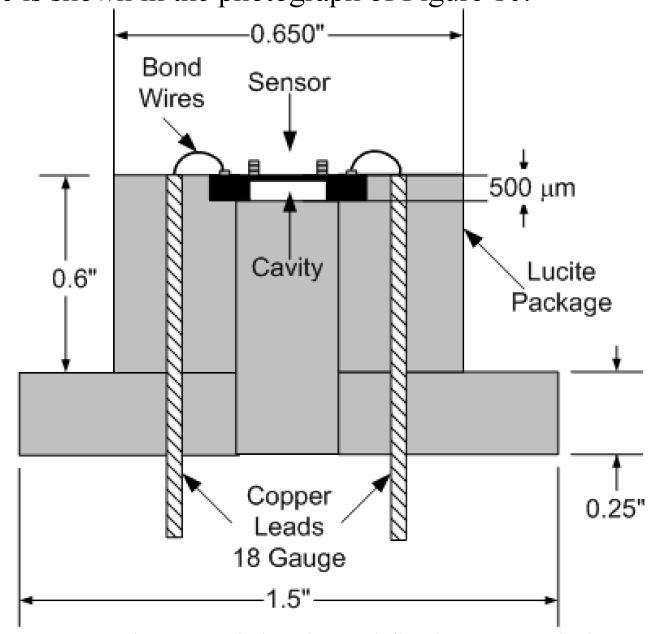

Figure 9: Lucite package with leads and flush mounted chip.

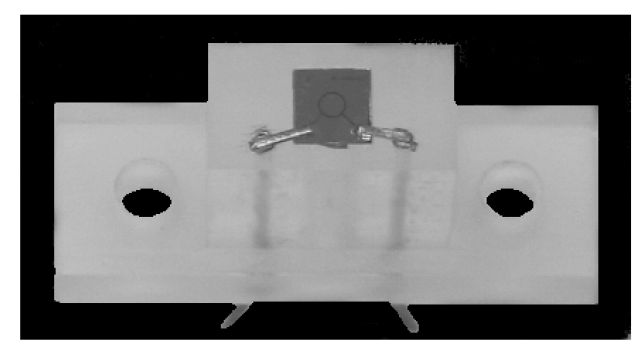

Figure 10: Optical photograph of packaged microphone.

\section{EXPERIMENTAL SETUP AND RESULTS}

Prior to full characterization of the device, the blocked electrical properties were measured prior to the release of the device using a vector impedance meter (HP Model 4294A) and are shown in Table 2.

Table 2: Measured blocked electrical properties.

\begin{tabular}{|c|c|c|c|}
\hline$C_{e b}[\mathrm{nF}]$ & $R_{e}[M \Omega]$ & $\varepsilon_{r}$ & $\tan \delta$ \\
\hline 13.9 & 1.7 & 809 & 0.007 \\
\hline
\end{tabular}

Following the electrical characterization, the packaged device was mounted at the end of a $2.54 \mathrm{~cm}$ square, normal incidence plane wave tube (Figure 11) to measure the frequency response and device linearity against a reference microphone (Brüel \& Kjær (B\&K) 1/8" Type 4138). The excitation signal was provided by a B\&K PULSE Multi-Analyzer System to a $50.8 \mathrm{~mm}$ coaxial compression driver (BMS Model 4590P) via a power amplifier (Techron Model 7540). The B\&K PULSE was also used for data acquisition.

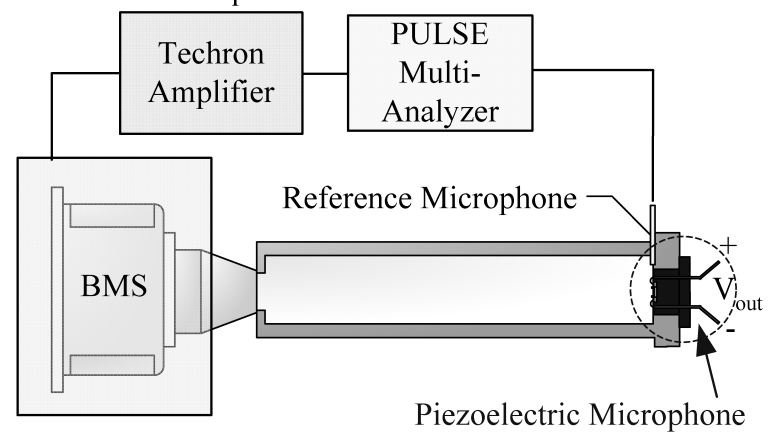

Figure 11: Experimental setup in a plane-wave tube.

The frequency response was measured using periodic random noise at $94 d B$ and 1000 spectral averages over a bandwidth from 0 to $6.4 \mathrm{kHz}$ with a $1 \mathrm{~Hz}$ bin width. A representative frequency response function of the sensor is shown in Figure 12 in terms of magnitude and phase. The response is flat over the testable frequency range up to $6.7 \mathrm{kHz}$, except for a low frequency roll-off of approximately $100 \mathrm{~Hz}$. This is most likely due to the vent resistance; however, the magnitude of this contribution is unknown, as the value for the vent resistance due to packaging leaks has not been measured.

Representative linearity results, shown in Figure 13, were taken at $1 \mathrm{kHz}$. For each measurement, 100 averages were taken of the rms output voltage from the measured power spectrum using a $1 \mathrm{~Hz}$ bin width with a rectangular window centered at $1 \mathrm{kHz}$ to avoid spectral leakage. The device is linear $\left(R^{2}=0.9995\right)$ up to (at least) the maximum testable sound pressure level of $169 \mathrm{~dB}$. 
The maximum testable level is limited by the output capacity of the compression driver. The measured sensitivity is $0.75 \mu \mathrm{V} / \mathrm{Pa}$.
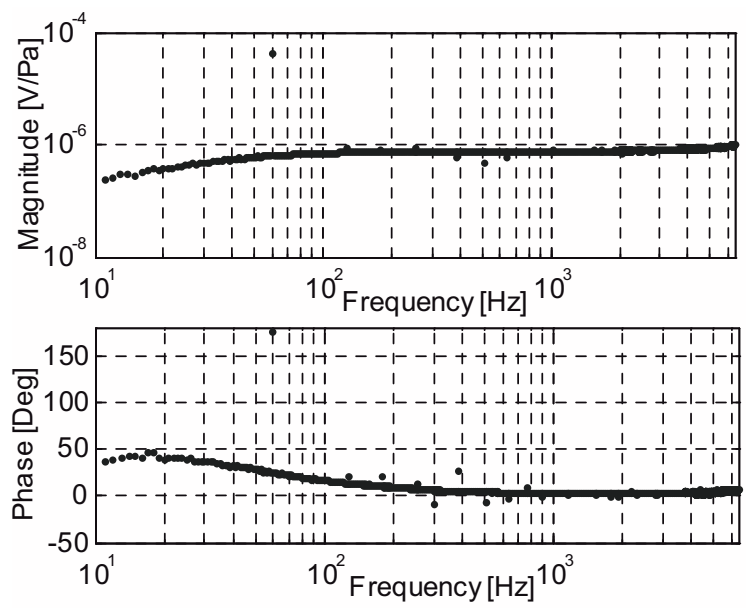

Figure 12: Measured sensor frequency response.

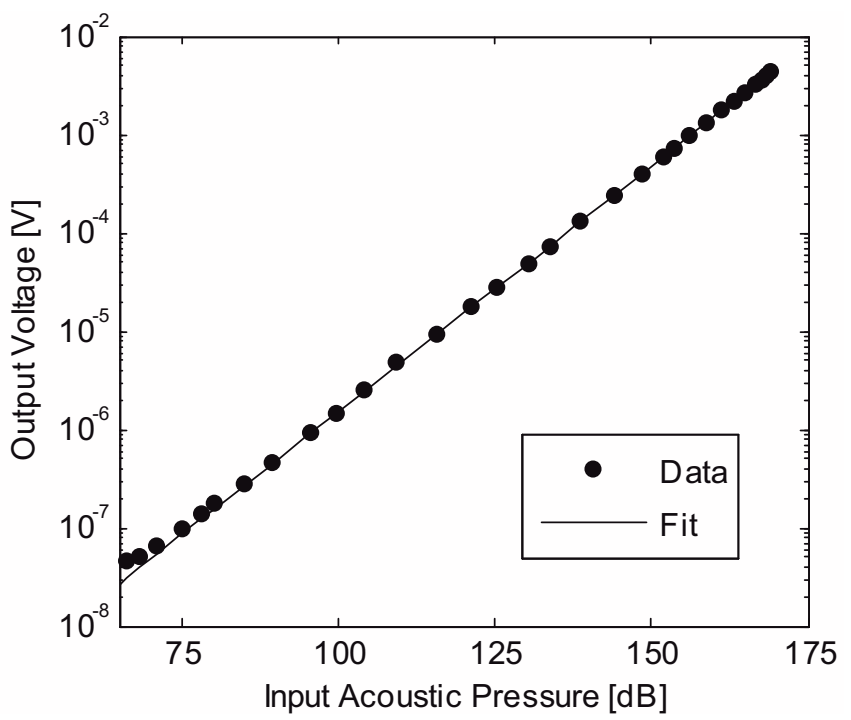

Figure 13: Measured sensor linearity at $1 \mathrm{kHz}$.

To obtain noise floor measurements, the sensor was mounted in a triple Faraday cage along with an SRS 560 low-noise preamplifier, through which the sensor output was routed, to tripleshield the sensor from potential electromagnetic interference. For the measurement, the preamplifier was set to a gain setting of 1000, with a high pass filter cutoff of $0.03 \mathrm{~Hz}$ and a low pass filter cutoff of $1 \mathrm{MHz}$. The output of the preamplifier was then routed, via feed-through adapters, out of the Faraday cage to an SRS 785 dynamic signal analyzer.

The noise floor spectrum of the sensor is shown in Figure 14 to $12.8 \mathrm{kHz}$, along with the noise spectrum due to the experimental setup alone. The noise spectrum of the experimental setup alone was obtained by shorting the inputs to the preamplifier and recording the resulting output voltage signal, using the same conditions and setup as the sensor noise floor measurement. This noise power spectral density is characteristic of a resistance shunted by a capacitance. The resistance in this case is the sensor dielectric resistance, $R_{e}$, and the capacitance is the blocked electrical capacitance, $C_{e b}$. The resistance and capacitance form a low-pass transfer function that spectrally shapes the thermal noise. When integrated over an infinite bandwidth, the resulting mean square noise voltage is given by $k T / C_{e b}$, where $k$ is Boltzmann's constant and $T$ is the ambient temperature. For this device, the low pass filter has a cutoff frequency of $6.7 \mathrm{~Hz}$, determined by $1 / 2 \pi R_{e} C_{e b}$.

There are three characteristic regions in this spectrum. Below $6.7 \mathrm{~Hz}$, there exists a relatively flat region where the noise is dominated by the resistance of the sensor. Then, from $6.7 \mathrm{~Hz}$ up to approximately $2 \mathrm{kHz}$ a $1 / f$ rolloff in the noise is observed that is consistent with a capacitive dominated noise source. Above $2 \mathrm{kHz}$, the noise spectrum flattens out again as the sensor noise approaches the setup noise. For a $1 \mathrm{~Hz}$ bin width centered at $1 \mathrm{kHz}$, the output voltage with no acoustic signal applied is $3.69 \mathrm{nV}$, which corresponds to an equivalent acoustic pressure of $4.93 \mathrm{mPa}$ or $47.8 \mathrm{~dB}$.

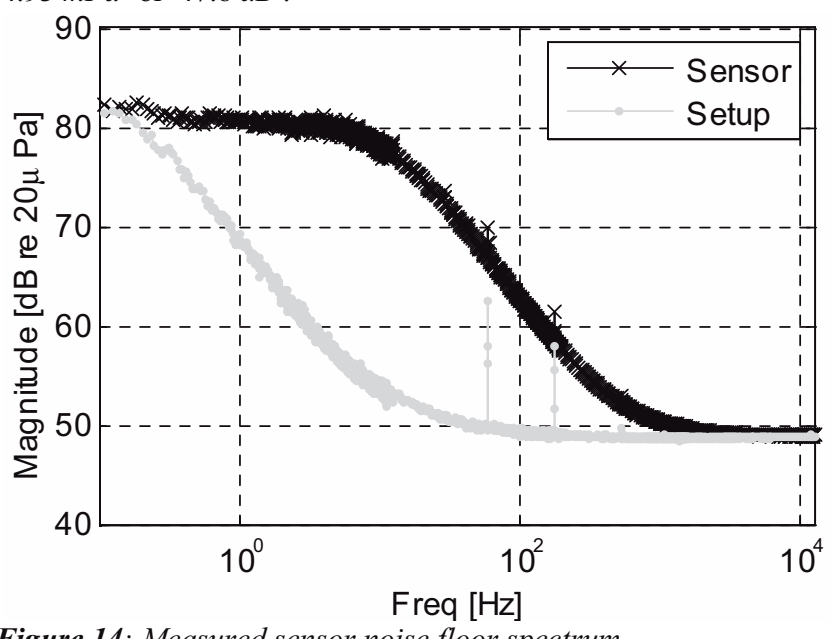

Figure 14: Measured sensor noise floor spectrum.

Because the device is a reciprocal transducer, the actuator frequency response can be used to gain additional information about the sensor response, such as the bandwidth [10]. In particular, the acoustically actuated frequency response could only be measured up to $6.7 \mathrm{kHz}$ due to testing limitations of the impedance tube. Using reciprocity, the bandwidth can be estimated via use of the electrically actuated frequency response as the piezoelectric microphone.

In order to determine the actuator frequency response, scanning laser vibrometry was performed. The packaged device was mounted under an Olympus BX60 microscope, with a 5x objective lens. The device was excited using white noise from an HP 33120A arbitrary waveform generator. Velocity data were obtained over the surface of the composite diaphragm using a Polytec OFV 3001S laser scanning vibrometer, with a Polytec OFV-074 microscope adapter. Diaphragm displacement was obtained via integration of the measured velocity frequency response. The actuator frequency response is shown in Figure 15 in terms of magnitude and phase. The resonance frequency is $50.8 \mathrm{kHz}$. This resonance provides an estimate of the usable bandwidth as a microphone.

The device characteristics are summarized in Table 3, demonstrating that this microphone has a larger bandwidth and dynamic range than existing piezoelectric microphones.

\section{CONCLUSIONS}

A MEMS-based piezoelectric microphone was developed for aeroacoustic measurements and consists of a circular composite 
annular ring of PZT for electromechanical transduction. An electroacoustic equivalent circuit model was used to design the device. Experimental characterization yielded a sensitivity of $0.75 \mu \mathrm{V} / \mathrm{Pa}$, with a linear dynamic range from $47.8 \mathrm{~dB}$ to at least $169 d B$. The resonant frequency was estimated to be $50.8 \mathrm{kHz}$ by measuring the actuator frequency response. The device characteristics are summarized in Table 3.

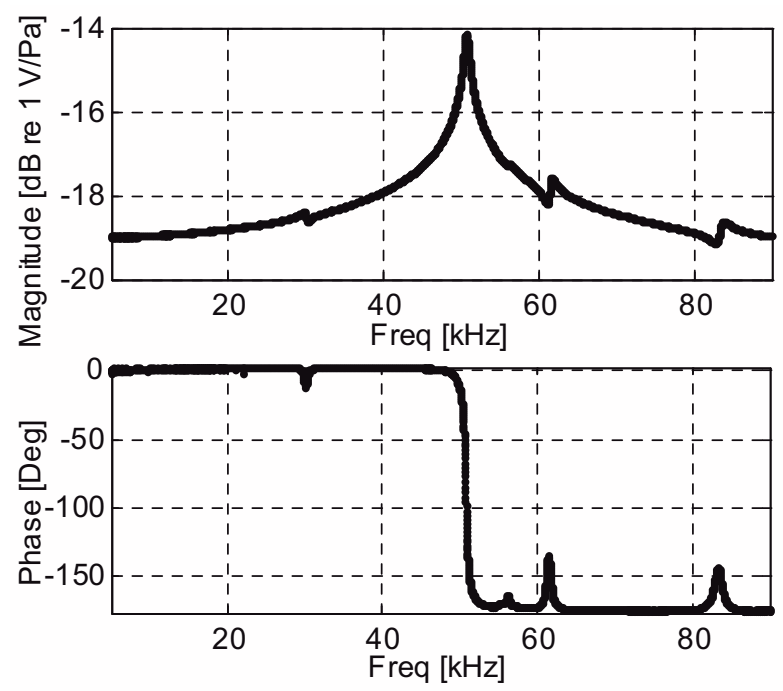

Figure 15: Measured actuator frequency response.

Table 3: Summary of experimental results and comparison to previous work.

\begin{tabular}{|l|c|c|c|c|}
\hline Researcher & Material & $\begin{array}{c}\text { Sensitivity } \\
{[\mathrm{mV} / \mathrm{Pa}]}\end{array}$ & $\begin{array}{c}f_{\text {res }} \\
{[\mathrm{kHz}]}\end{array}$ & $\begin{array}{c}\text { Noise Floor } \\
{[\mathrm{dB}]}\end{array}$ \\
\hline Present Work & $\mathrm{PZT}$ & $0.75 \mu \mathrm{V} / \mathrm{Pa}$ & 50.8 & 47.8 \\
\hline Kim et al. $[\mathbf{4}]$ & $\mathrm{ZnO}$ & 1 & 16 & $50^{1}$ \\
\hline Ried et al. $[\mathbf{5}]$ & $\mathrm{ZnO}$ & 0.92 & 18 & $57^{1}$ \\
\hline Lee et al. $[\mathbf{2}]$ & $\mathrm{ZnO}$ & 30 & 0.89 & $\mathrm{~N} / \mathrm{R}$ \\
\hline Royer et al. $[\mathbf{3}]$ & $\mathrm{ZnO}$ & 0.25 & 10 & $66.02^{2}$ \\
\hline Schellin et al. $[\mathbf{6}]$ & polyurea & $0.004-0.030$ & $\mathrm{~N} / \mathrm{R}$ & $\mathrm{N} / \mathrm{R}$ \\
\hline Ko et al. $[\mathbf{8}]$ & $\mathrm{ZnO}$ & $0.51^{3}$ & 7.3 & $\mathrm{~N} / \mathrm{R}$ \\
\hline
\end{tabular}

${ }^{1}$ A-weighted, ${ }^{2}$ Method not reported, ${ }^{3}$ At resonance

The theoretical predictions do not match the experimental results. Specifically, the predicted sensitivity was roughly a factor of three larger than that measured. This discrepancy is due to a combination of factors. First, there tends to be large uncertainties in the thin-film material properties. More importantly, the devices were statically deflected on the order of the diaphragm thickness due to large in-plane compressive stresses from the $\mathrm{TiO}_{2}$. These large static deflections render the small-deflection assumption in the composite plate theory invalid.

Future work includes the extension of the composite plate model to large deflections. These models will then be validated against the current design and then subsequently used to synthesize an optimal design A new package design is necessary to provide a vent channel to the microphone for a controlled low frequency rolloff. Further characterization of the microphone is needed in order to determine variability from device to device, and to investigate reliability issues such as fatigue and stability of the piezoelectric modulus.

\section{ACKNOWLEDGMENTS}

Financial support for this research was provided by Sandia National Laboratories and monitored by Kent Pfeifer. The authors are also grateful for significant fabrication assistance provided by Stephanie Jones of Sandia National Laboratories.

\section{REFERENCES}

[1] T. Mueller, ed. Aeroacoustic Measurements. 2002, SpringerVerlag: Berlin. 313.

[2] S. Lee, R. Ried, and R. White, "Piezoelectric Cantilever Microphone and Microspeaker". Journal of Microelectromechanical Systems, 1996. 5,4: p. 238-42.

[3] M. Royer, J. Holmen, M. Wurm, and O. Aadland, "ZnO on Si Integrated Acoustic Sensor". Sensors and Actuators, A: Physical, 1983. 4: p. 357-62.

[4] E. Kim, J. Kim, and R. Muller. "Improved IC-Compatible Piezoelectric Microphone and CMOS Process".Int Conf Solid State Sens Actuators. San Francisco, CA. IEEE. (1991), p. 270-3.

[5] R. Ried, E. Kim, D. Hong, and R. Muller, "Piezoelectric Microphone with On-Chip CMOS Circuits". Journal of Microelectromechanical Systems, 1993. 2,3: p. 111-20.

[6] R. Schellin, G. Hess, W. Kuehnel, G. Sessler, and E. Fukada. "Silicon Subminiature Microphones with Organic Piezoelectric Layers: Fabrication and Acoustical Behaviour".7th International Symposium on Electrets. Berlin, IEEE. (1991), pp. 929-34.

[7] P. Scheeper, A. van der Donk, W. Olthuis, and P. Bergveld, "A Review of Silicon Microphones". Sensors and Actuators A, 1994. 44,1: p. 1-11.

[8] S. Ko, Y. Kim, S. Lee, S. Choi, and S. Kim, "Micromachined Piezoelectric Membrane Acoustic Device". Sensors and Actuators, A: Physical, 2003. 103: p. 130-4.

[9] E. Kim, R. Muller, and P. Gray. "Integrated Microphone with CMOS Circuits on a Single Chip".International Electron Devices Meeting. Washington, D.C. IEEE. (1989), p. 880-3.

[10] S.D. Senturia, Microsystems Design. 2001, Kluwer Academic Publishers, Inc. Boston, chapter 5.

[11] G. Wang, B. Sankar, L. Cattafesta, and M. Sheplak. "Analysis of a Composite Piezoelectric Circular Plate with Initial Stresses for MEMS".International Mechanical Engineering Congress \& Exposition. New Orleans, LA. ASME. (2002).

[12] N. Setter, ed. Piezoelectric Materials in Devices. 2002, N. Setter, EPFL Swiss Federal Institute of Technology: Lausanne.

[13] D. Blackstock, Fundamentals of Physical Acoustics. 2000, John Wiley \& Sons, Inc. p. 153-6.

[14] B. Tuttle, J. Voigt, T. Headley, B. Potter, D. Dimos, R. Schwartz, M. Dugger, J. Michael, R. Nasby, T. Garino, and D. Goodnow, "Ferrolectric Thin Film Microstructure Development and Related Property Enhancement". Ferroelectrics, 1994. 151: p. 11-20. 